\title{
PRONOSTICO INMEDIATO DE TORMENTAS CONVECTIVAS POR RADAR - UNA ACTUALIZACION
}

\author{
SADIEL NOVO \\ Centro de Física Atmosférica, Instituto de Meteorología \\ Loma de Casablanca, Municipio Regla, Ciudad Habana, Cuba \\ Apartado 17032, Habana 17. C.P. 11700, Cuba \\ Email: sadiel.novo@insmet.cu
}

Recibido Agosto 2006 - Aceptado Marzo 2007

\begin{abstract}
RESUMEN
Una de las principales aplicaciones del radar meteorológico es el pronóstico inmediato (decenas de minutos a unas pocas horas de antelación) de los eventos de precipitación convectiva. Esto reviste gran importancia pues es precisamente en este lapso de tiempo donde son menos efectivos los pronósticos numéricos. Se repasan brevemente algunas de las técnicas de pronóstico inmediato reportadas en los últimos años, haciendo énfasis en aquellas que emplean solamente información de un radar convencional. Se distingue entre las que pronostican el movimiento de las tormentas como un todo y aquellas que permiten distinguir su movimiento interno, señalando sus ventajas y desventajas en situaciones meteorológicas concretas. Como ejemplo se presentan, para un caso de estudio escogido, aplicaciones simples de dos de estas técnicas. Por último se discute la utilidad de la técnica del sistema de referencia móvil para obtener las componentes del viento no observadas a partir de un solo radar Doppler o incluso un radar convencional, y sus implicaciones para la obtención de magnitudes termodinámicas.
\end{abstract}

Palabras clave: radar meteorológico, tormenta convectiva, pronóstico inmediato, Doppler

\begin{abstract}
NOWCASTING OF CONVECTIVE STORMS BY RADAR - AN ACTUALIZATION
\end{abstract}

One of the main applications of the meteorological radar is the nowcasting (some tens of minutes to some hours forward) of convective precipitation events. It has a great importance since this is the space of time in which the numerical forecasting is less effective. Some of the nowcasting techniques reported in the last years are reviewed shortly, making emphasis in those that use only information from a conventional radar. A distinction is made among those that predict the total movement of the storms and those that allow distinguishing their internal movement, pointing out their advantages and disadvantages in concrete meteorological situations. It is presented, for a chosen case of study, simple applications of two of these techniques. Finally, the utility of the moving frame of reference technique for obtaining the not observed wind components starting from a single Doppler radar or even a conventional one, and its implications in the retrieval of thermodynamic magnitudes, is discussed.

Keywords: radar, convective storm, nowcasting, Doppler

\section{INTRODUCCIÓN}

La definición de "pronóstico inmediato" o "nowcasting" ha evolucionado a través de los años. Browning (1982) dice que es una combinación de descripción del tiempo presente más extrapolación de sus tendencias hasta 2 horas hacia delante. En Conway (1998) se interpreta como el pronóstico detallado hasta 6 horas, con refinamientos de hasta 12 horas, haciéndose cada vez más borrosa su distinción del pronóstico a muy corto plazo.

A pesar de los grandes avances de las dos últimas décadas en la predicción numérica operacional del tiempo, es muy probable que el pronóstico inmediato mantenga un importante rol en el futuro inmediato de la vigilancia meteorológica (Li \& Lai, 2004). Esto se debe a la poca habilidad de la modelación numérica en las primeras horas, producto principalmente del 
tiempo requerido para el procesamiento, análisis y asimilación de gran cantidad de datos, más el tiempo consumido propiamente en la simulación. La tendencia de las técnicas de nowcasting es la de incorporar, además del pronóstico del movimiento y evolución de las tormentas, la predicción cuantitativa de la cantidad de precipitación (Wilson et al, 1998).

Las herramientas primarias para detectar tormentas convectivas son los radares del tiempo, los detectores de rayos y los satélites meteorológicos. Los primeros son los más empleados históricamente para estos fines y entre sus principales ventajas se encuentra la alta resolución espacial y temporal de sus datos, además de permitir la observación tridimensional directa de su objetivo.

En situaciones de desarrollo convectivo, el problema del pronóstico involucra la iniciación, evolución y movimiento de las tormentas. El pronóstico de iniciación se ha desarrollado fundamentalmente a partir del monitoreo del movimiento relativo entre las líneas de convergencia de la capa fronteriza y las nubes cúmulos cercanas, con ayuda de radares Doppler de aire claro y datos de satélite (Wilson et al, 1998). También la simulación numérica ha contribuido mucho en determinar, por ejemplo, la importancia de la cizalladura del viento de bajo nivel normal a la línea de convergencia.

Una vez que existan tormentas en el área de interés es sumamente importante, tanto desde el punto de vista operacional como científico, contar con un procedimiento objetivo para detectarlas, analizarlas y seguir su evolución. El algoritmo de identificación y seguimiento debe mantener un compromiso entre sencillez y robustez. La simplicidad disminuirá considerablemente el tiempo de corrida (siendo ideal el trabajo en tiempo real) y la robustez redundará en un mejor análisis y pronóstico.

Debe tenerse en cuenta que las tormentas individuales (celdas simples) poseen un tiempo de vida cercano a los 20 min (Wilson et al, 1998), se mueven según el viento medio y son capaces de unirse para formar grupos de tormentas (con un tiempo de vida mucho mayor y movimiento muy diferente al de las celdas simples), que pueden a su vez subdividirse con posterioridad. También resulta muy útil determinar el campo de viento interno de las tormentas, pues esto permite discernir las zonas más peligrosas (zonas de fuerte convergencia/fuerte vorticidad vertical) y llevarse una mejor idea de su estructura (zonas de crecimiento/disipación) y movimiento (zonas de propagación).

El presente trabajo expone brevemente algunas de las técnicas de pronóstico inmediato reportadas en los últimos 10 años, haciendo énfasis en aquellas que emplean solamente información de un radar convencional. Se distingue entre las que pronostican el movimiento de las tormentas como un todo y aquellas que permiten distinguir su movimiento interno. Se discute además la utilidad de la técnica del sistema de referencia móvil para obtener las componentes del viento no observadas a partir de un solo radar Doppler o incluso un radar convencional, y sus implicaciones para la obtención de magnitudes termodinámicas. Los objetivos propuestos son: 1- Actualizar el conocimiento de la temática en nuestro país, 2- Investigar cuál técnica se adecúa más a un interés particular mediante la discusión de casos meteorológicos concretos.

\section{TÉNICAS DE PRONÓSTICO INMEDIATO}

Las primeras técnicas para el pronóstico inmediato de la posición de tormentas, con radares convencionales, se desarrollaron a comienzos de los años 60 mediante la extrapolación de ecos de radar (Wilson et al, 1998). En ellas se correlacionaban dos patrones digitales de ecos sucesivos y se determinaba el vector de movimiento en la dirección en que se obtuviera la máxima correlación. Luego se extrapolaba linealmente el campo total de eco según este vector suponiendo que no ocurrían cambios en su tamaño o intensidad. Estudios realizados durante esta misma década demostraron la importancia de tener en cuenta la escala de tamaño del fenómeno convectivo analizado a la hora de extrapolar su evolución. La exactitud de estos pronósticos a menudo disminuye rápidamente durante los primeros 30 minutos debido al corto tiempo de vida de las celdas convectivas individuales. No obstante, sistemas más organizados como líneas de tormentas y superceldas pueden ser satisfactoriamente extrapolados durante mayores períodos de tiempo.

Rinehart \& Garvey (1978) fueron de los primeros en obtener patrones de movimiento interno dentro de tormentas, mediante el análisis de correlación cruzada entre pequeñas regiones del campo de reflectividad en instantes sucesivos. Ellos denominaron su método TREC (siglas de Tracking Radar Echoes by Correlation). La comparación con datos de radar Doppler mostró grandes analogías, así como algunas diferencias que serán discutidas posteriormente en este trabajo.

También en los años 70 surgieron los primeros algoritmos para seguir celdas individuales en dos dimensiones mediante técnicas de reconocimiento de patrones (Wilson et al, 1998). Estas se basaron en el seguimiento y pronóstico de las áreas, los centroides pesados u otros parámetros de los contornos de radar cerrados que se asumían como representativos de las tormentas convectivas individuales. Se agruparon bajo el nombre de "técnicas de seguimiento del centroide". Desde finales de esta década y las siguientes (años 80 y 90) se prestó especial atención al tratamiento de las tormentas como entidades tridimensionales (e.g., Crane 1979 y Rosenfeld 1987), al posible empleo de las tendencias de tamaño e intensidad del eco (e.g., Tsonis \& Austin 1981) para el pronóstico inmediato, así como a la inclusión de 
las fusiones (mergers) y las divisiones (splits) naturales entre tormentas (e.g., Rosenfeld 1987, Witt \& Johnson 1993, Dixon \& Wiener 1993). El uso más completo de la información disponible permitió contar con una poderosa herramienta para el análisis científico de las tormentas.

Para una revisión más detallada de las técnicas de nowcasting hasta mediados de los años 90, consultar Wilson et al (1998).

\section{MOVIMIENTO TOTAL DE TORMENTAS}

Hasta 1995 los sistemas de pronóstico inmediato por radar más empleados usaban para la identificación de tormentas un solo umbral de reflectividad. Un ejemplo fue el llamado TITAN (siglas de Thunderstorm Identification, Tracking, Analysis, and Nowcasting) (Dixon \& Wiener, 1993) en el cual se identificaba como tormenta, en cada patrón de eco digital, cualquier grupo tridimensional de pixels con reflectividad mayor que cierto valor umbral y volumen mayor que cierto otro umbral, siempre que estuvieran unidos entre sí por al menos uno de sus lados. Para el seguimiento, TITAN aproximaba cada tormenta por una elipse ajustada al área proyectada por la tormenta sobre un plano horizontal, y luego aplicaba un procedimiento de optimización basado en las diferencias de posición y volumen para determinar las parejas de tormentas (padre e hija) entre dos barridos consecutivos. Las fusiones y las divisiones se determinaban por simple lógica geométrica.

En 1998 se publicó (Johnson et al, 1998) la nueva versión del algoritmo de identificación y seguimiento empleado por la red de radares Doppler de Estados Unidos (WSR-88D). En él se definieron 7 umbrales de reflectividad en orden descendente para la identificación de tormentas, lo cual mejoró considerablemente esta labor, sobre todo en líneas o grupos compactos de tormentas donde pueden haber varias celdas embebidas en un contorno de alta reflectividad. Para el seguimiento se empleó la técnica de seguimiento del centroide volumétrico pesado por la reflectividad. Una primera aproximación para la posición de una celda que se venía siguiendo se hallaba mediante su vector de movimiento obtenido en el barrido previo. En caso de una celda surgida en un determinado barrido, se empleaba el promedio de todos los vectores de movimiento de las celdas en el barrido anterior o un vector velocidad entrado por el usuario. Como posición de una celda en el último barrido se hallaba aquella cuya distancia a la posición de primera aproximación fuera mínima. El vector de movimiento entre barridos se hallaba uniendo con una línea recta las posiciones en los barridos previo y último. El vector de movimiento definitivo de la celda se obtenía mediante un ajuste lineal mínimo cuadrático de las posiciones de los centroides en los diez últimos barridos realizados por el radar.

\section{TRACE3D}

Handwerker (2002) ideó otro algoritmo automático para el pronóstico inmediato de tormentas convectivas con solo datos de reflectividad. Para la identificación se determinan primeramente los volúmenes barridos por el haz del radar que tengan reflectividad mayor que determinado umbral. Luego se halla el valor máximo de reflectividad dentro de cada uno de estos volúmenes. A continuación se definen las celdas de tormenta como aquellas regiones contiguas, dentro de cada volumen, con reflectividad mayor que la diferencia entre la máxima y cierto valor fijo. Para el seguimiento se emplean las velocidades de las celdas durante los barridos anteriores a fin de extrapolar la posición de una celda individual al barrido más reciente, y luego se buscan sucesores de esta celda en la vecindad de la posición predicha de manera semejante a como hacía SCIT (siglas de Storm Cell Identification and Tracking). Sin embargo, TRACE3D emplea un procedimiento más sofisticado para detectar las fusiones y divisiones de las celdas.

\section{APLICACIÓN SIMPLE}

La Figura 1 muestra la salida gráfica de un programa escrito en Matlab para identificar y seguir ecos de radar de forma muy simple. El caso mostrado corresponde al análisis de 6 observaciones de radar (MRL-5, $1=10 \mathrm{~cm}$ ) obtenidas el 23 de Julio del 2001 en Camagüey, entre 4:50 y 5:21 pm. Este día fue reportado un tornado cerca de las 5 pm muy cerca de la posición de radar (indicada por una cruz hacia el centro de la figura) hacia el SE. La distancia máxima de observación del radar fue de $180 \mathrm{~km}$ y solo se observa la imagen CAPPI a 3 $\mathrm{km}$ de altura correspondiente a la última observación, con la identificación y el seguimiento superpuestos.

Las condiciones ambientales prevalecientes este día y un análisis por radar de la tormenta tornádica han sido descritos por Novo et al (2004). El mapa sinóptico presentó la dorsal del anticiclón de verano del Atlántico cubriendo todo el territorio cubano, lo cual propició que sobre Camagüey prevalecieran las altas presiones con bajo gradiente, provocando un fuerte calentamiento en la tarde. Una onda tropical surcó los mares al sur de la región oriental de Cuba durante este día. El sondeo de la 1:00 pm sobre Camagüey mostró alta inestabilidad atmosférica con valores de energía potencial convectiva disponible y del índice de ascenso de $5954 \mathrm{~J} / \mathrm{kg}$ y $-7.9^{\circ} \mathrm{C}$, respectivamente. $\mathrm{La}$ tormenta donde se produjo el tornado reportado se movió entre W y WSW, manteniendo valores de reflectividad por encima de $50 \mathrm{dBZ}$ durante $90 \mathrm{~min}$, con topes nubosos por encima de los 12 $\mathrm{km}$ sin poder medir valores mayores debido a la cercanía de la tormenta al radar.de imágenes CAPPI a baja altura, empleando la técnica de etiquetado de componentes conectados (Pitas, 2000). 


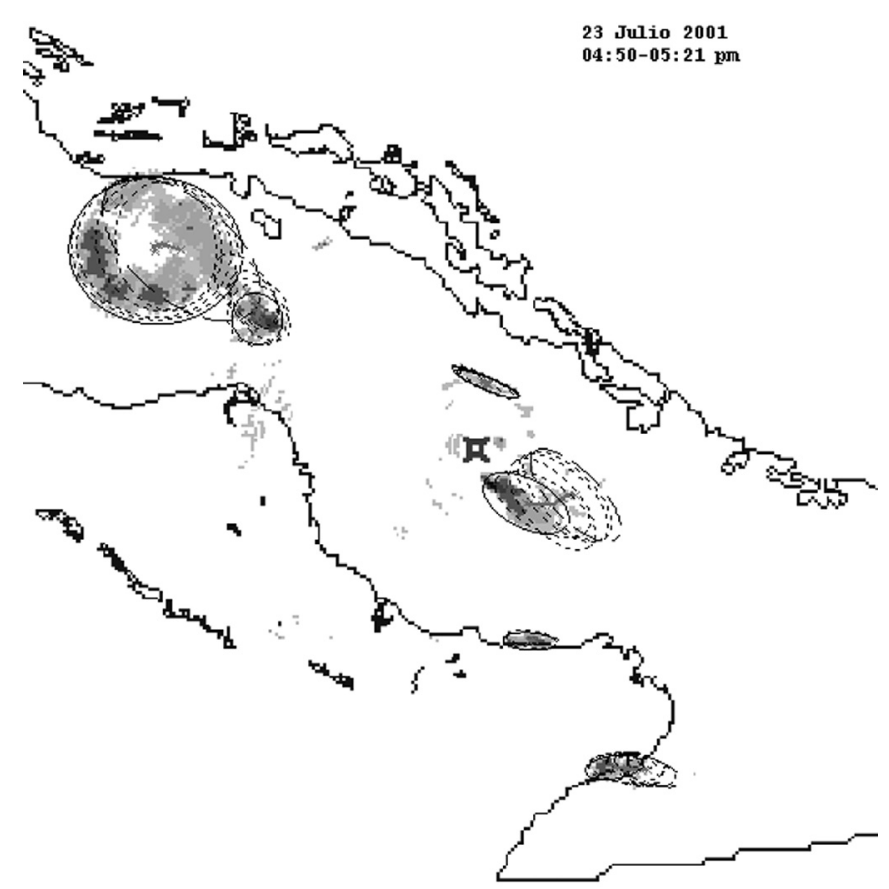

Figura 1: Identificación y seguimiento de tormentas sobre Camagüey, Cuba.

La identificación se realizó en dos dimensiones, a partir Se definió una tormenta como cualquier grupo de pixels, unidos por al menos un lado, cuyas reflectividades exceden los $10 \mathrm{dBZ}$. También se empleó un umbral de área para desechar objetos pequeños. Cada tormenta fue representada por una elipse de iguales momentos centrales normalizados de segundo orden que su área, y fue ubicada a partir del centro de esta elipse. De esta manera se obtuvo una descripción general de su forma y orientación. Para el seguimiento de una tormenta específica se buscó en la imagen siguiente, entre todos los centros calculados, aquel cuya distancia a la posición de la tormenta en cuestión fuera mínima. Luego se compararon las correspondientes tormentas (padre e hija) en cuanto a su área y posición y si ambas diferencias no superaron ciertos umbrales, se asociaron sus posiciones como consecutivas de la misma tormenta y se trazó una flecha entre ambas para indicar su desplazamiento.

La Figura 2 muestra una ampliación de la tormenta que produjo el tornado. La cruz en la esquina superior izquierda representa la posición del radar. Las elipses en líneas discontinua y continua representan, respectivamente, las posiciones inicial y final de la tormenta, mientras que las posiciones intermedias se representan con líneas punteadas. Nótese el desplazamiento hacia el W y SW de la tormenta. El fichero de salida incluyó, para cada instante, el área, la posición del centro, la reflectividad (Z) máxima, la $\mathrm{Z}$ promedio y la suma total de $\mathrm{Z}$ para cada tormenta presente.
La aplicación descrita adolece de varias cosas. La primera es que emplea un solo umbral de $\mathrm{Z}$ para la identificación, por lo que no permite discernir tormentas pequeñas embebidas en clusters. La siguiente es que solo permite obtener propiedades bidimensionales, perdiendo la importante información tridimensional sobre el eco. Por último, el método de seguimiento es demasiado simple. No obstante, ha mostrado cierta habilidad para tomar en cuenta las fusiones y divisiones entre tormentas, así como el surgimiento y disipación de celdas vecinas (Novo, 2007).

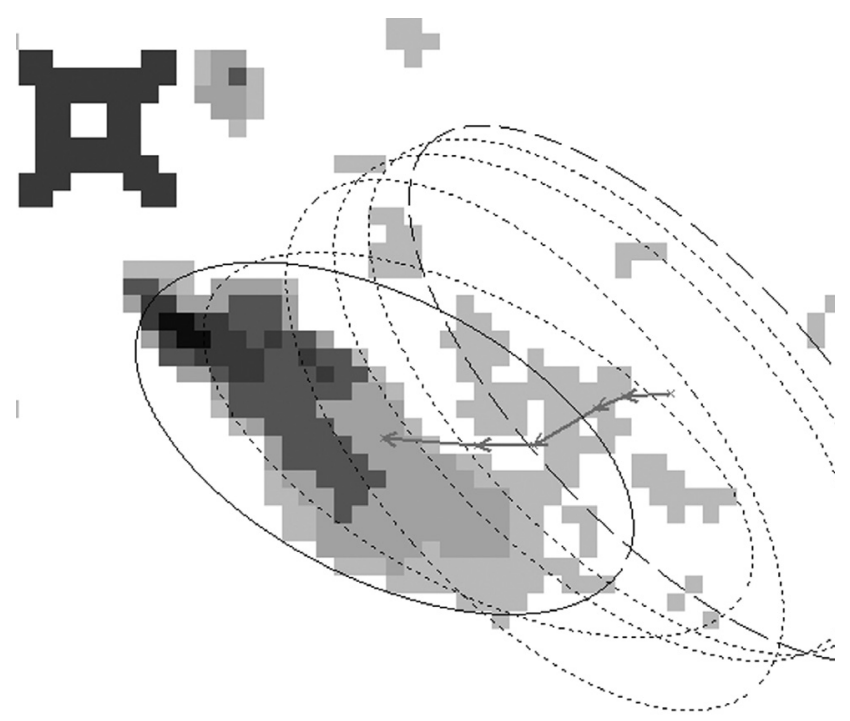

Figura 2: Ampliación de la tormenta tornádica.

\section{MOVIMIENTO INTERNO DE TORMENTAS}

Tradicionalmente el movimiento interno de celdas de tormenta se ha obtenido mediante radares Doppler, preferiblemente más de uno. Sin embargo, la mayoría de las veces solo se cuenta con datos de un solo radar que puede incluso no ser Doppler; éste es el caso de Cuba. Debido a la ventaja de los radares Doppler de medir directamente la componente radial de la velocidad de un grupo de hidrometeoros, hasta mediados de los años 90 se había prestado poca atención al desarrollo de esquemas objetivos para determinar la estructura cinemática interna de tormentas con un radar convencional. Uno de los primeros intentos fue el llamado método TREC (Rinehart \& Garvey, 1978). En él se buscaba la correlación máxima entre pequeñas regiones del eco de precipitación (arreglos de pixels) para dos observaciones sucesivas de radar (dos PPI o dos CAPPI a igual inclinación o altura, respectivamente). Luego se trazaban vectores de movimiento entre los dos centros de las zonas que más correlacionaban. De esta forma se obtenía un campo vectorial bidimensional de velocidades semejante al obtenido con radares Doppler, el cual se extrapolaba para el pronóstico inmediato de la posición del eco. 
Este método fue luego aplicado satisfactoriamente por Smythe \& Zrnic (1983) y Tuttle \& Foote (1990) para datos de aire claro.

Los vectores de movimiento obtenidos por el método TREC representan el flujo de aire, en la región barrida por el radar, solo en caso de ecos de aire claro (Tuttle \& Foote, 1990). Para ecos de precipitación, dichos vectores representan el flujo de aire en el nivel superior donde fue generado el patrón lluvioso (Rinehart \& Garvey, 1978), a diferencia del caso Doppler donde se mide directamente la velocidad del aire en el área examinada. Esto genera alguna discrepancia con el campo obtenido por radares Doppler.

\section{COTREC}

Los vectores TREC a menudo son afectados por ecos no meteorológicos o sombras en el haz del radar, y por variaciones rápidas de pequeña escala dentro del patrón de radar. Esto se traduce en vectores de velocidad erróneos. Para atenuar estos efectos, Li et al (1995) propusieron forzar los vectores obtenidos por TREC a cumplir con la ecuación de continuidad en dos dimensiones, de modo que se suavizara el campo de velocidades. Esto no significa que se conserve la reflectividad, pero mejora mucho los resultados. Aplicaron el nuevo método, al que llamaron COTREC (siglas de COntinuity of TREC), a diferentes tipos de tormentas y en regiones de tanto precipitación convectiva como estratiforme, con buenos resultados.

La obtención precisa de vectores de movimiento interno por la técnica COTREC, permitió la identificación cuantitativa de las zonas de crecimiento y disipación dentro de tormentas. Para ello se calculó la diferencia en reflectividad media entre las pequeñas zonas de cada barrido enlazadas por los vectores de movimiento obtenidos. Las áreas de valores positivos de esta diferencia se identificaron como regiones de intensificación del eco, mientras las áreas de valores negativos indicaron regiones de disipación. Además, COTREC fue aplicado con bastante éxito al caso de tormentas severas. El ploteo de los vectores COTREC en un sistema coordenado relativo a la propia tormenta analizada, permitió identificar las zonas de vorticidad ciclónica en el caso de una supercelda. Mecklenburg (2000) optimizó este método para el nowcasting de precipitación (tanto convectiva como estratiforme) en una región de gran orografía, incorporando el suavizamiento temporal de los datos y restringiendo la cantidad de arreglos a correlacionar.

La obtención precisa de vectores de movimiento interno por la técnica COTREC, permitió la identificación cuantitativa de las zonas de crecimiento y disipación dentro de tormentas. Para ello se calculó la diferencia en reflectividad media entre las pequeñas zonas de cada barrido enlazadas por los vectores de movimiento obtenidos. Las áreas de valores positivos de esta diferencia se identificaron como regiones de intensificación del eco, mientras las áreas de valores negativos indicaron regiones de disipación. Además, COTREC fue aplicado con bastante éxito al caso de tormentas severas. El ploteo de los vectores COTREC en un sistema coordenado relativo a la propia tormenta analizada, permitió identificar las zonas de vorticidad ciclónica en el caso de una supercelda. Mecklenburg (2000) optimizó este método para el nowcasting de precipitación (tanto convectiva como estratiforme) en una región de gran orografía, incorporando el suavizamiento temporal de los datos y restringiendo la cantidad de arreglos a correlacionar.

\section{SWIRLS}

El Observatorio de Hong Kong ha desarrollado un sistema de nowcasting llamado SWIRLS (siglas de ShortRange Warnings of Intense Rainstorms in Localized Systems), que produce pronósticos cuantitativos de lluvia de hasta 3 horas de antelación (Li \& Lai, 2004a). Este sistema se basa en la extrapolación de ecos de radar usando la técnica TREC discutida anteriormente, con algunas mejoras adicionales, como son chequeos de consistencia (comparación de la dirección de un vector con la media de sus vecinos) y filtrado por el método de análisis objetivo de Cressman (1959). Escogiendo adecuadamente el tamaño de los arreglos de pixels a correlacionar, los vectores de movimiento obtenidos se emplean satisfactoriamente para monitorear y extrapolar el movimiento de prácticamente cualquier tipo de tormenta moderadamente intensa, desde celdas convectivas individuales hasta líneas o grupos de tormentas y superceldas. Para una resolución de 480x480 pixels en el campo de reflectividad que se obtiene del radar, emplean arreglos de 19x19 pixels separados una distancia de 5 pixels (Li \& Lai, 2004b).

\section{CICLONES TROPICALES}

La aplicación con ligeras modificaciones de la técnica TREC a la obtención del campo de viento horizontal en ciclones tropicales, ha dado muy buenos resultados (Tuttle \& Gall, 1995, 1999; Lai, 1999). El predominio del movimiento advectivo sobre el movimiento vertical de los hidrometeoros en estos sistemas es crucial. Lai (1999) usó un esq uema de integración semilagrangiano para producir patrones de reflectividad pronóstico a partir de un campo de vectores TREC. El paso de tiempo fue de 6 minutos. Tuttle \& Gall (1999) sustituyeron la componente radial de los vectores TREC obtenidos, por los valores Doppler medidos por su radar, mejorando así el campo de velocidades para el pronóstico.

\section{APLICACIÓN SIMPLE}



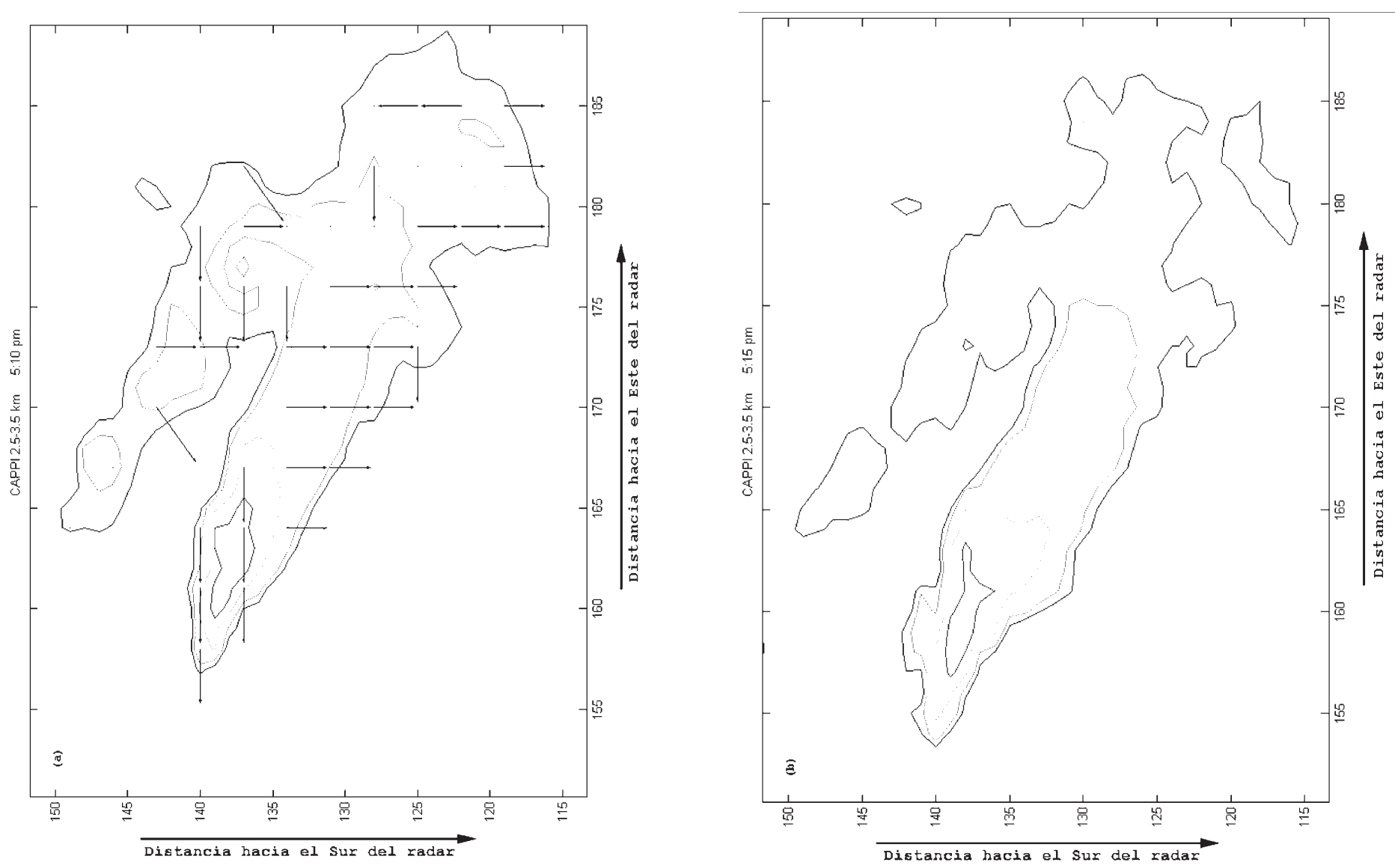

Figura 3: Aplicación del método TREC (CAPPI, 3 km) en su forma más simple.

La Figura 3 muestra el resultado de aplicar el método TREC, en su forma más simple, a los instantes (a) 5:10 pm y (b) 5:15 pm de la tormenta tornádica del 23 de Julio del 2001.

Los contornos indican isolíneas de 10, 20, 30, 40 y 50 $\mathrm{dBZ}$, comenzando por el borde exterior de la tormenta. Las flechas en la imagen (a) indican movimientos horizontales internos en la tormenta, correspondientes a arreglos de 9x9 pixels (valores de Z), separados por 3 pixels entre sí. Los puntos indican que la mayor correlación se encontró con el mismo arreglo de puntos en ambas imágenes. El radio de búsqueda para la correlación solo tuvo en cuenta los 8 vecinos más próximos a cualquier punto. No se ha aplicado ningún método de suavizamiento al campo vectorial obtenido. La posición del radar estuvo en el punto $(150,150)$, al NW de la tormenta. Nótese la mayor densidad de flechas en las regiones más intensas de la tormenta, indicando mayor movimiento horizontal. También el movimiento hacia el $\mathrm{W}$ de la región de mayor gradiente de reflectividad dentro de la parte más intensa, mientras que la región de menor gradiente (al sur) se mueve hacia el Sur. Esto provoca un alargamiento de la parte más intensa de la tormenta en la dirección NW-SE.

\section{OBTENCIÓN DE COMPONENTES DE VIENTO NO OBSERVADAS CON UN SOLO RADAR DOPPLER}

Hasta comienzos de los años 90, los métodos que empleaban datos de un solo radar Doppler para obtener información del campo de viento en tres dimensiones, no hacían un uso completo de la información disponible. Uno de ellos fue el llamado VAD (Velocity-Azimuth Display) (Lhermitte \& Atlas, 1961; Browning \& Wexler, 1968), en el cual se procesaban mediciones de velocidad radial de los hidrometeoros contra ángulo azimutal para diferentes ángulos de elevación, con el fin de obtener perfiles verticales de velocidad, divergencia (suponiendo conocida la velocidad terminal de caída de los hidrometeoros) y deformación horizontal media del viento. Integrando la ecuación de continuidad podía obtenerse la velocidad vertical media del viento, con los errores intrínsecos a este método producto de lo difícil de contar con una buena condición de frontera superior en cualquier capa de aire (O'Brien, 1970; Matejka \& Bartels, 1998). Una gran desventaja del método es que solo se aplica bien a casos de precipitación 
estratiforme (campos de viento cuasilineales en la horizontal) y bajos ángulos de elevación.

Posteriormente Waldteufel \& Corbin (1979) demostraron que las técnicas que presuponían cumplida una hipótesis de linealidad para el campo de viento horizontal, como VAD y VARD (Velocity-Area Display) (Easterbroock, 1975), eran casos particulares de un método más general que llamaron VVP (Volume-Velocity Processing). Otra generalización aplicable en casos de precipitación estratiforme fue la desarrollada por Srivastava et al (1986) y Matejka \& Srivastava (1991), llamada EVAD (Extended Velocity-Azimuth Display), en la cual no se requería conocer la velocidad terminal de caída de los hidrometeoros (sino que se estimaba) y podía usar datos de altos ángulos de elevación. La aplicación de algoritmos para compensar errores en los datos le permitió adaptarse mejor a situaciones de fuerte cizalladura vertical del viento o campos de viento no uniformes en la horizontal, produciendo mejores resultados en casos reales.

Ninguno de los métodos anteriores combina los datos de velocidad radial con datos de reflectividad para estimar el campo de viento. Otros como TREC/COTREC, donde se asume que la reflectividad se conserva entre barridos (lo cual casi nunca es cierto), emplean restricciones muy fuertes y por tanto no tienen en cuenta toda la variabilidad incluida en las mediciones. Todo esto conlleva a que no pueda obtenerse un campo tridimensional de velocidades de alta precisión con un solo radar Doppler por alguno de estos métodos.

La técnica del sistema de referencia móvil, sugerida por Gal-Chen (1982) y desarrollada por Gal-Chen \& Zhang (1993), Laroche \& Zawadzki (1994), Shapiro et al (1995), Zhang \& Gal-Chen (1996), Liou (1999, 2001, 2002) y Lazarus (1999, 2001), consiste básicamente en emplear para la estimación un sistema coordenado, que no tiene que estar fijo a la Tierra, para el cual la variación temporal de la reflectividad en el volumen observado es la más pequeña. Esto es equivalente a encontrar las componentes óptimas de velocidad que advectan el campo de reflectividad mientras se incumple menos la conservación de su magnitud. Para esto solo se necesitan datos de reflectividad de al menos dos barridos de radar en tiempos cercanos. Una vez encontrados dichos valores, se emplean los datos de reflectividad $\mathrm{y}$ velocidad radial junto a varias restricciones impuestas al sistema, para obtener las componentes de perturbación del campo de viento mediante un método variacional. Cada componente del campo de viento real se halla sumando la componente de advección y la de perturbación correspondiente.

En el método original de Gal-Chen (1982) y Gal-Chen \& Zhang (1993) se empleaban solo dos restricciones débiles en el cálculo de las componentes de perturbación, a saber, la conservación lagrangiana de la reflectividad y la ecuación que relaciona la velocidad radial a sus componentes cartesianas.
Zhang \& Gal-Chen (1996) introdujeron pesos adecuados a estas dos restricciones para evitar errores por diferencia de escala entre ellas, mejorando la exactitud del método. También el suavizamiento temporal y espacial adecuado contribuyó a la mejoría. Liou (1999) agregó otras dos restricciones a la determinación de las componentes de perturbación: conservación de la masa para reducir la divergencia y pequeña vorticidad vertical para suavizar y así reducir el ruido del campo obtenido. Con esto, mejoró considerablemente la obtención de las componentes horizontales del viento en bajos niveles para datos de radar simulados. Suponiendo un estimado grosero para la divergencia horizontal y eliminando la restricción que tenía en cuenta la velocidad radial, se pudo incluso obtener de forma cualitativamente bien, dichas componentes con solo datos simulados de reflectividad. La obtención del campo de velocidad vertical fue menos satisfactoria, aunque cualitativamente consistente con el campo real simulado. Esto se debió a que solo se tomó para el cálculo una capa horizontal de datos. Para mejorar el viento vertical, en dicho trabajo se sugiere obtenerlo a partir de componenentes horizontales obtenidas con suficiente exactitud, o aplicar el método de obtención del viento en una capa a varios planos verticales.

Lazarus et al (2001) aplicaron el método de Zhang \& Gal-Chen (1996) a datos reales de convección profunda para obtener el campo de viento total. Al igual que Liou (1999), obtuvieron el viento vertical con baja calidad cuantitativa. Para mejorarlo se valieron de la ecuación de continuidad (ya sugerida por Liou) y de la alta correlación entre velocidad radial y velocidad vertical en capas medias y altas, producto de la cercanía de la tormenta al radar. Liou \& Luo (2001) aplicaron el método de Liou (1999) a datos reales y demostraron que la magnitud del viento azimutal obtenido está siempre por debajo del valor real, sobre todo cuando esta componente es mucho mayor que la radial. Esto significa que la componente azimutal obtenida depende de la posición del radar, siendo de más baja calidad cuando el viento se dirige más perpendicular al haz del radar. Liou (2002) demostró que dicho error en la componente azimutal ocurre cuando el tiempo entre barridos (resolución temporal) es mayor que el cociente entre la resolución espacial del radar y la velocidad del fenómeno observado, lo cual se cumple en la mayoría de los casos. No obstante se demuestra, para un caso real, que bajo la suposición de que la resolución temporal es menor que la mitad del cociente entre la longitud de onda y la velocidad del fenómeno observado (típicamente $10 \mathrm{~km}$ y $20 \mathrm{~m} / \mathrm{s}$, respectivamente), puede obtenerse un sistema móvil optimizado sobre el cual se simplifica considerablemente el error en la obtención de la componente azimutal de perturbación. Esta condición puede cumplirse bien en los radares Doppler de hoy con resoluciones temporales menores de $5 \mathrm{~min}$. Una vez estimado el campo de viento en tres dimensiones con suficiente 
resolución, podemos derivarlo en el tiempo y obtener los campos de presión y temperatura mediante el ajuste, por alguna técnica variacional, de las ecuaciones horizontales y vertical de movimiento a los datos obtenidos, pudiendo emplearse restricciones adicionales como son una ecuación termodinámica (Weygandt, 2002; Liou et al, 2003) o comparación con campos obtenidos por simulación numérica (Liou et al, 1991; Gao et al, 2001).

\section{SUMARIO Y CONCLUSIONES}

Hasta aquí se han discutido algunas de las técnicas de pronóstico inmediato de tormentas por radar reportadas en los últimos años, sobre todo las que emplean solamente información de un radar convencional. Aquellas que pronostican el movimiento como un todo se basan en técnicas de identificación y seguimiento de patrones en los ecos de radar (contornos, centroides en tres dimensiones, etc) y permiten calcular y seguir la evolución de las propiedades de cada tormenta individual. Tener en cuenta las fusiones y divisiones entre tormentas, aunque sea de una manera sencilla, mejora mucho el pronóstico. Pueden obtenerse mapas bidimensionales de viento interno, así como zonas de crecimiento/disipación con datos de reflectividad, solamente si se suavizan adecuadamente los mapas vectoriales obtenidos. En caso de contar con datos de velocidad radial de un radar Doppler puede mejorarse sustancialmente la obtención del campo horizontal de velocidades, e incluso estimar adecuadamente la componente vertical del viento, siempre que se planifiquen estrategias de observación adecuadas y se minimicen al máximo los errores inherentes al método de medición discreta del radar.

\section{REFERENCIAS}

Browning, K. A. Nowcasting. Academic Press, 1982. 256 p.

Browning, K. A.; Wexler, R. The determination of kinematic properties of a wind field using Doppler radar. Journal of Applied Meteorology, v. 7, p. 105-113, 1968.

Conway, B. J. An overview of nowcasting techniques. In: SAF Training Workshop - Nowcasting and very short range forecasting, EUMETSAT, 1998, p. 34-43.

Cressman, G. P. An operational objective analysis system. Monthly Weather Review, v. 87, p. 367-374, 1959.

Dixon, M.; Wiener, G. TITAN: thunderstorm identification, tracking, analysis, and nowcasting - a radar based methodology. Journal of Atmospheric and Oceanic Technology, v. 10, p. 785-797, 1993.

Easterbrook, C. C. Estimating horizontal wind fields by two dimensional curve fitting of single-Doppler radar measurements. In: 16th Radar Meteorology Conference, Houston, 1975, p. 214-219.
Gal-Chen, T. Errors in fixed and moving frame of references: Application for conventional and Doppler radar analysis. Journal of Atmospheric Sciences, v. 39, p. 2279-2300, 1982.

Gal-Chen, T.; Zhang, J. On the optimal use of reflectivities and single Doppler radar velocities to deduce 3D motions. In: 26th International Conference on Radar Meteorology, Oklahoma, 1993, p. 414-416.

Gao, J.; Xue, M.; Shapiro, A.; Xu, Q.; Droegemeier, K. Three-dimensional simple adjoint velocity retrievals from single-Doppler radar. Journal of Atmospheric and Oceanic Technology, v. 18, n. 1, p. 26-38, 2001.

Handwerker, J. Cell tracking with TRACE3D - a new algorithm. Atmospheric Research, v. 61, p. 15-34, 2002.

Johnson, J. T.; MacKeen, P. L.; Witt, A.; Mitchell, E. D.; Stumpf, G. J.; Eilts, M. D.; Thomas, K. W. The storm cell identification and tracking algorithm: an enhanced WRS88D algorithm. Weather and Forecasting, v. 13, p. 263-276, 1998.

Lai, E. S. T. TREC application in tropical cyclone observation. ESCAP/WMO Typhoon Committee Annual Review, p. 135-139, 1999.

Laroche, S.; Zawadzki, I. A variational analysis method for retrieval of three-dimensional wind field from singleDoppler radar data. Journal of the Atmospheric Sciences, v. 51, n. 18, p. 2664-2682, 1994.

Lazarus, S.; Shapiro, A.; Droegemeier, K. Analysis of the GalChen-Zhang single-Doppler velocity retrieval. Journal of the Atmospheric and Oceanic Technology, v. 16, p. 5-18, 1999.

Lazarus, S.; Shapiro, A.; Droegemeier, K. Application of the Zhang-Gal-Chen single-Doppler velocity retrieval to a deepconvective storm. Journal of the Atmospheric Sciences, v. 58, p. 998-1016, 2001.

Lhermitte, R.M.; Atlas, D. Precipitation motion by pulse Doppler. In: 9th Weather Radar Conference, Kansas, 1961, p. 218-223.

Li, L.; Schmid, W,; Joss, J. Nowcasting of motion and growth of precipitation with radar over a complex orography. Journal of Applied Meteorology, v. 34, p. 1286-1300, 1995.

Li, P. W.; Lai, E. S. T. Applications of radar-based nowcasting techniques for mesoscale weather forecasting in Hong Kong. Meteorological Applications, v. 11, p. 253-264, 2004a.

Li, P. W.; Lai, E. S. T. Short-range quantitative precipitation forecasting in Hong Kong. Journal of Hydrology, v. 288, p. 189-209, 2004b.

Liou, Y. C.; Gal-Chen, T.; Lilly, D. K. Retrieval of wind temperature and pressure from single-Doppler radar and a numerical model. In: 25th International Conference on Radar Meteorology, Paris, 1991, p. 151-154. 
Liou, Y. C. Single radar recovery of cross-beam wind components using a modified moving-frame of reference technique. Journal of Atmospheric and Oceanic Technology, v. 16, p. 1003-1016, 1999.

Liou, Y. C.; Luo, I. S. An investigation of the moving frame single-Doppler wind retrieval technique using Taiwan Area Mesoscale Experiment low-level data. Journal of Applied Meteorology, v. 40, p. 1900-1917, 2001.

Liou, Y. C. An explanation of the wind speed underestimation obtained from a least squares type single-Doppler radar velocity retrieval method. Journal of Applied Meteorology, v. 41, p. 811-823, 2002.

Liou, Y. C. A three-dimensional variational approach for deriving the thermodynamic structure using Doppler wind observations -An application to a subtropical squall line. Journal of Applied Meteorology, v. 42, p. 1443-1454, 2003.

Matejka, T.; Srivastava, R. C. An improved version of the Extended Velocity-Azimuth Display analysis of singleDoppler radar data. Journal of Atmospheric and Oceanic Technology, v. 8, n. 4, p. 453-466, 1991.

Matejka, T.; Bartels, D. L. The accuracy of vertical air velocities from Doppler radar data. Monthly Weather Review, v. 126, p. 92-117, 1998.

Mecklenburg, S.; Joss, J.; Schmid, W. Improving the nowcasting of precipitation in an Alpine region with an enhanced radar echo tracking algorithm. Journal of Hydrology, v. 239, p. 46-68, 2000

Novo, S.; Martínez, D.; Gamboa, F. Estudio por radar de dos tormentas convectivas observadas sobre Camagüey, Cuba: tormenta tornádica del 23 de Julio del 2001. En: I Taller de Meteorología Tropical, Convención Trópico, La Habana, 2004.

Novo, S. Seguimiento automático de tormentas convectivas por radar. Enviado para su publicación en este mismo número de la Revista Brasileira de Meteorología, 2007.

O'Brien, J. J. Alternative solutions to the classical vertical velocity problem. Journal of Applied Meteorology, v. 9, n. 2, p. 197-203, 1970.

Pitas, I. Digital image processing: algorithms and applications. Ed. John Wiley \& Songs, 2000. 419 p.

Rinehart, R.E.; Garvey, E.T. Three-dimensional storm motion detection by conventional weather radar. Nature 273, p. 287-289, 1978.

Rosenfeld, D. Objective method for analysis and tracking of convective cells as seen by radar. Journal of Atmospheric and Oceanic Technology, v. 4, p. 422-434, 1987.

Shapiro, A.; Ellis, S.; Shaw, J. Single-Doppler velocity retrievals with Phoenix II data: Clear air and microburst wind retrievals in the planetary boundary layer. Journal of the Atmospheric
Sciences, v. 52, n. 9, p. 1265-1287, 1995.

Smythe, G. R.; Zrnic, D. S. Correlation analysis of Doppler radar data and retrieval of the horizontal wind. Journal of Climate and Applied Meteorology, v. 22, p. 297-311, 1983.

Srivastava, R. C. et al. Doppler radar study of the trailing anvil region associated with a squall line. Journal of the Atmospheric Sciences, v. 43, p. 356-377, 1986.

Tsonis, A. A.; Austin, G. L. An evaluation of extrapolation techniques for the short-term prediction of rain amounts. Atmosphere and Ocean, v. 19, p. 54-65, 1981.

Tuttle, J. D.; Foote, G. B. Determination of the boundary layer airflow from a single Doppler radar. Journal of Atmospheric and Oceanic Technology, v. 7, p. 218-232, 1990.

Tuttle, J. D.; Gall, R. L. Radar analysis of hurricanes Andrew and Hugo. In: 21 th Conference on Hurricanes and Tropical Meteorology, Miami, 1995, p. 608-610.

Tuttle, J. D.; Gall, R. L. A single-radar technique for estimating the winds in tropical cyclones. Bulletin of the American Meteorological Society, v. 80, p. 653-668, 1999.

Waldteufel, P.; Corbin, H. On the analysis of single-Doppler radar data. Journal of Applied Meteorology, v. 18, p. 532542, 1979.

Weygandt, S. S.; Shapiro, A.; Droegemeier, K. K. Retrieval of model initial fields from single-Doppler observations of a supercell thunderstorm (2 partes). Monthly Weather Review, v. 130, p. 433-476, 2002.

Wilson, J. W.; Crook, N. A.; Mueller, C. K.; Sun, J.; Dixon, M. Nowcasting thunderstorms: a status report. Bulletin of the American Meteorological Society, v. 79, p. 2079-2099, 1998.

Witt, A.; Johnson, J. T. An enhanced storm cell identification and tracking algorithm. In: 26th International Conference on Radar Meteorology, Oklahoma, 1993, p. 514-521.

Zhang, J.; Gal-Chen, T. Single-Doppler wind retrieval in the moving frame of reference. Journal of the Atmospheric Sciences, v. 53, n. 18, p. 2609-2623, 1996.

Li, L.; Schmid, W,; Joss, J. Nowcasting of motion and growth of precipitation with radar over a complex orography. Journal of Applied Meteorology, v. 34, p. 1286-1300, 1995.

Li, P. W.; Lai, E. S. T. Applications of radar-based nowcasting techniques for mesoscale weather forecasting in Hong Kong. Meteorological Applications, v. 11, p. 253-264, 2004a.

Li, P. W.; Lai, E. S. T. Short-range quantitative precipitation forecasting in Hong Kong. Journal of Hydrology, v. 288, p. 189-209, 2004b.

Liou, Y. C.; Gal-Chen, T.; Lilly, D. K. Retrieval of wind temperature and pressure from single-Doppler radar and a numerical model. In: 25th International Conference on Radar Meteorology, Paris, 1991, p. 151-154.

Liou, Y. C. Single radar recovery of cross-beam wind 
components using a modified moving-frame of reference technique. Journal of Atmospheric and Oceanic Technology, v. 16, p. 1003-1016, 1999.

Liou, Y. C.; Luo, I. S. An investigation of the moving frame single-Doppler wind retrieval technique using Taiwan Area Mesoscale Experiment low-level data. Journal of Applied Meteorology, v. 40, p. 1900-1917, 2001.

Liou, Y. C. An explanation of the wind speed underestimation obtained from a least squares type single-Doppler radar velocity retrieval method. Journal of Applied Meteorology, v. 41, p. 811-823, 2002.

Liou, Y. C. A three-dimensional variational approach for deriving the thermodynamic structure using Doppler wind observations -An application to a subtropical squall line. Journal of Applied Meteorology, v. 42, p. 1443-1454, 2003.

Matejka, T.; Srivastava, R. C. An improved version of the Extended Velocity-Azimuth Display analysis of singleDoppler radar data. Journal of Atmospheric and Oceanic Technology, v. 8, n. 4, p. 453-466, 1991.

Matejka, T.; Bartels, D. L. The accuracy of vertical air velocities from Doppler radar data. Monthly Weather Review, v. 126, p. 92-117, 1998.

Mecklenburg, S.; Joss, J.; Schmid, W. Improving the nowcasting of precipitation in an Alpine region with an enhanced radar echo tracking algorithm. Journal of Hydrology, v. 239, p. 46-68, 2000.

Novo, S.; Martínez, D.; Gamboa, F. Estudio por radar de dos tormentas convectivas observadas sobre Camagüey, Cuba: tormenta tornádica del 23 de Julio del 2001. En: I Taller de Meteorología Tropical, Convención Trópico, La Habana, 2004.

Novo, S. Seguimiento automático de tormentas convectivas por radar. Enviado para su publicación en este mismo número de la Revista Brasileira de Meteorología, 2007.

O'Brien, J. J. Alternative solutions to the classical vertical velocity problem. Journal of Applied Meteorology, v. 9, n. 2, p. 197-203, 1970.

Pitas, I. Digital image processing: algorithms and applications. Ed. John Wiley \& Songs, 2000. 419 p.

Rinehart, R.E.; Garvey, E.T. Three-dimensional storm motion detection by conventional weather radar. Nature 273, p. 287-289, 1978.
Rosenfeld, D. Objective method for analysis and tracking of convective cells as seen by radar. Journal of Atmospheric and Oceanic Technology, v. 4, p. 422-434, 1987.

Shapiro, A.; Ellis, S.; Shaw, J. Single-Doppler velocity retrievals with Phoenix II data: Clear air and microburst wind retrievals in the planetary boundary layer. Journal of the Atmospheric Sciences, v. 52, n. 9, p. 1265-1287, 1995.

Smythe, G. R.; Zrnic, D. S. Correlation analysis of Doppler radar data and retrieval of the horizontal wind. Journal of Climate and Applied Meteorology, v. 22, p. 297-311, 1983.

Srivastava, R. C. et al. Doppler radar study of the trailing anvil region associated with a squall line. Journal of the Atmospheric Sciences, v. 43, p. 356-377, 1986.

Tsonis, A. A.; Austin, G. L. An evaluation of extrapolation techniques for the short-term prediction of rain amounts. Atmosphere and Ocean, v. 19, p. 54-65, 1981.

Tuttle, J. D.; Foote, G. B. Determination of the boundary layer airflow from a single Doppler radar. Journal of Atmospheric and Oceanic Technology, v. 7, p. 218-232, 1990.

Tuttle, J. D.; Gall, R. L. Radar analysis of hurricanes Andrew and Hugo. In: 21 th Conference on Hurricanes and Tropical Meteorology, Miami, 1995, p. 608-610.

Tuttle, J. D.; Gall, R. L. A single-radar technique for estimating the winds in tropical cyclones. Bulletin of the American Meteorological Society, v. 80, p. 653-668, 1999.

Waldteufel, P.; Corbin, H. On the analysis of single-Doppler radar data. Journal of Applied Meteorology, v. 18, p. 532$542,1979$.

Weygandt, S. S.; Shapiro, A.; Droegemeier, K. K. Retrieval of model initial fields from single-Doppler observations of a supercell thunderstorm (2 partes). Monthly Weather Review, v. 130, p. 433-476, 2002.

Wilson, J. W.; Crook, N. A.; Mueller, C. K.; Sun, J.; Dixon, M. Nowcasting thunderstorms: a status report. Bulletin of the American Meteorological Society, v. 79, p. 2079-2099, 1998.

Witt, A.; Johnson, J. T. An enhanced storm cell identification and tracking algorithm. In: 26th International Conference on Radar Meteorology, Oklahoma, 1993, p. 514-521.

Zhang, J.; Gal-Chen, T. Single-Doppler wind retrieval in the moving frame of reference. Journal of the Atmospheric Sciences, v. 53, n. 18, p. 2609-2623, 1996. 